\title{
Implicaciones epistemológicas en la formación de profesores de Educación Física: mirada desde los alumnos
}

\author{
Epistemological implications in physical education teacher training: a view from students' perspective
}

\author{
Alberto Calderón García
}

\begin{abstract}
Resumen
Muchas variables inciden en la formación del profesorado en educación física, pues el proceso de registro calificado y acreditación de calidad suscita el debate interno entre académicos que sustentan los currículos, para definir el objeto de estudio, perfil ocupacional, perfil profesional y competencias de las diferentes carreras. Aumenta la tensión la aparición de los exámenes de competencias académicas de la educación superior, ECAES, que reflejan los enfoques curriculares y, seleccionadas por la comunidad académica, son administrados por los organismos estatales para evaluar a los estudiantes de últimos semestres, basados en referencias internacionales.

En esas circunstancias y asumiendo que las competencias son una alternativa para fortalecer el desarrollo humano y el pensamiento autónomo, surge en esta investigación y la pregunta por cómo formamos a los licenciados en educación física en las instituciones de educación superior. Al establecer las relaciones epistemológicas con diferentes variables pedagógicas y los campos: educación física, deportes y recreación, los estudiantes del Programa Curricular de Licenciatura en Educación Física de la Universidad Pedagógica Nacional, como sujetos conocedores, se involucraron en la interpretación de la información, su significado y sus valores interactuando con el investigador y adoptando la investigación como praxis.
\end{abstract}

Palabras clave: Competencias, acreditación, formación de profesores, currículos, pedagogía, concepciones, estudiantes, educación física, deporte, recreación.

\section{Abstract}

The training of physical education teachers is influenced by many factors. The process of qualified registration and quality accreditation arouses an internal discussion among scholars supporting the curricula, as they try to define the object of study, occupational and professional profiles, as well as the competences of each career. The tension is increased by the ECAES (Exam of Academic Competences on Higher Education), that reflect the curricular focuses and -selected by the academic community- are administered by the State entities to evaluate senior students having as reference the international standards.

In this context, and assuming competences as an alternative to strengthen both human development autonomous thinking, the main question of this research arouses: How are higher education colleges training the Physical education teachers? When establishing the epistemological relationships with different pedagogical variables and physical education, sports, and recreation fields, the students of the Physical Education Program at the Universidad Pedagógica Nacional, as expert subjects, got involved in data analysis, its meaning and values, interacting with the researcher and assuming the research as a practice.

\section{Key words: $\quad$ Competence, accreditation, teacher training, curriculum, pedagogy, notion, students, physical education, sport, recreation.}

Fecha de recepción: 18 de octubre de 2007.

Fecha de aceptación: 9 de noviembre de 2007

* Profesor de la Universidad Pedagógica Nacional. Licenciado en Educación Física. Ph. D en Educación Física de la Universidad del Estado de la Florida. Autor de El deporte: de la autonomía a la hegemonía (2007). acalderon@pedagogica.edu.co 


\section{Introducción}

En Colombia muchas variables inciden, en la formación del profesorado en educación física y de profesionales del deporte, la recreación, la actividad física, la cultura fisica, etc., pues los procesos de registro calificado, autoevaluación y acreditación de calidad a que están sometidos los programas de instituciones de educación superior sirven, según Álvarez (2003), para que cada facultad "se esculque un poco y se encuentre con propuestas de renovación interesantes, con dinamización de procesos y creación de condiciones para el encuentro, la discusión y la autocrítica".

Ese proceso que promueve la calidad de la educación superior y la ampliación de cobertura también ha hecho emerger tensiones y debates internos sobre la docencia, investigación y extensión entre los académicos que sustentan los programas, tratando de definir el objeto de estudio, perfil ocupacional, perfil profesional y competencias de las diferentes carreras.

Así, algunos programas de educación física tienen como objeto de estudio el ser humano en movimiento, el desarrollo humano, la experiencia corporal, la motricidad humana, etc. En los programas de ciencias aplicadas al deporte o entrenamiento deportivo se estudia una variedad de objetos, como el rendimiento deportivo, el desempeño humano, el hombre en competencia, la formación deportiva, el deporte social, etc. Igual situación acontece en los programas que se apropian del campo de la recreación, que acuden a determinar su objeto de estudio como educar para el tiempo libre, el ser humano creativo, el ocio humano, etc. Otros, como los de cultura física y actividad física, presentan objetos de estudio similares a los anteriores o priorizan el logro deportivo o la salud y el estilo de vida, respectivamente.

Además de la urgencia que precisan los procesos mencionados, la comunidad académica empezó a discutir los Exámenes de Estado de Calidad de la Educación Superior, ECAES. Estos últimos supuestamente deben reflejar los paradigmas de la profesión, sus constructos teóricos y estructuras curriculares con sus planes de estudio, líneas de investigación, tendencias, perfil profesional y ocupacional, etc., para que basados en referencias internacionales cumplan el propósito de evaluar las competencias de los estudiantes de últimos semestres. El Ministerio de Educación Nacional las considera acciones constructivas o procesos cognitivos del individuo que le permiten desarrollar "conceptos, y generalizaciones, habilidades y destrezas, actitudes y valores y hábitos cognitivos" para desempeñarse adecuadamente durante su existencia.

Esto implicó una revisión epistemológica de los especialistas convocados por el Icfes para consensuar en lo atinente a las habilidades, conocimientos y valores que el estudiantado debe poseer al finalizar su carrera, y que aplicará en los contextos de su profesión, desarrollando competencias como las investigativas, administrativas, de comprensión de lectura, pedagógicas y didácticas en ciencias biológicas, y ciencias humanas, entrenamiento deportivo, etc.

Los resultados que debemos interpretar y analizar en las instituciones de educación superior, a pesar, de la probable derogación del decreto 1781, merecen un estudio con la influencia de Bahamón (2007), que califica las competencias desde una perspectiva pedagógica y sociohumanística y como una alternativa para fortalecer el desarrollo humano y el pensamiento autónomo, de modo que contribuyan a mejorar la calidad de vida de la persona. Sin embargo, destaca para utilizar las competencias como estrategia evaluativa, se tiene que revisar el marco conceptual, reorientar el currículo y la metodología, y construir ambientes de aprendizaje que promuevan la reflexión, la participación activa y crítica del estudiante en el proceso educativo.

Quiceno (2006) indica que en esta nueva sociedad del conocimiento se requiere cambiar la formación del sujeto, que enseña y del que aprende. Según este autor, la formación implica la capacidad de pensar y no la capacidad de actuar, y debe ser continua. Añade que el currículo se debe transformar y pasar de pensar en programas entendidos como unidades autónomas, a programas con cambios continuos: diseño modular de los programas, que demandan varias competencias organizadas en unidades que puedan certificarse una a una.

La normativa y jurídisprudencia que en varias ocasiones no representan el sentir ni el pensar de los académicos incidió en alto grado en el debate polisémico y multiteórico relacionado con las competencias y la formación de profesores y profesionales. La Ley 181 de 1995 definió, luego de una gran polémica y las motivaciones consabidas, la educación física, el deporte y la recreación, referenciados por los programas de los diferentes niveles escolares a partir de la Ley General de Educación (115 de 1994), en sus fines, objetivos y áreas obligatorias. Surgieron entonces intereses en las escuelas y colegios para abordar como área de su currículo la educación física el deporte y la recreación, dando lugar a cierto deportivismo en su desarrollo (Calderón, 2007). 
En varias universidades se ofreció un programa de licenciatura con mayor ponderación en el área de deportes; en otras los currículos privilegiaron una visión epistemológica constructivista y discursiva sobre el cuerpo. Incluso el decreto 272 de 1998 , que reglamentó la acreditación previa de programas en educación, produjo la oferta de programas de formación de profesores por niveles. Consecuencia de ello fue la proliferación de programas de licenciatura en básica primaria con énfasis en educación física, deporte y recreación.

\section{Planteamiento del problema}

Con este panorama es importante preguntarse cómo estamos formando a los licenciados y profesionales de educación física en las instituciones locales, departamentales y nacionales frente al contexto global, que de acuerdo con el CNA, el ICFES y el CONACES, debe orientar gran parte del desarrollo de los currículos terciarios en nuestro medio.

Como que las universidades en este proceso, deben realizar estudios endógenos que permitan aproximarse a la realidad y a la prospectiva. Con este trabajo se pretende establecer las relaciones epistemológicas generadas en los diferentes programas entre el estudiante como sujeto conocedor, el objeto de conocimiento y el contexto donde se construye, se aprende y se pone en práctica el conocimiento humano, para confrontarlos frente a otras epistemologías en diferentes latitudes.

Para abordar ese análisis se puede afirmar que en el país se han logrado aportes importantes como los Lineamientos Curriculares en Educación Física, Recreación y Deportes del Ministerio de Educación Nacional (2000), que presenta las diferentes tendencias de la Educación Física, cuyos énfasis en su "puesta en práctica en la escuela", son:

- Enseñanza y práctica del deporte y la condición física Actividades recreativas y de tiempo libre.

- Psicomotricidad.

- Estética corporal; mantenimiento de la forma y la salud.

Expresión corporal, danzas y representaciones artísticas.

Esas tendencias teórico-prácticas se fundamentan en la interacción de diferentes disciplinas científicas, como las ciencias biológicas y las ciencias sociales y humanas, que aún circulan en programas universitarios constituyendo su objeto de estudio, con base en autores foráneos primordialmente, como se observa en el cuadro siguiente, retomado de los lineamientos mencionados.

\begin{tabular}{ll} 
El cuerpo y el juego & \multicolumn{1}{c}{ Autor } \\
El hombre en movimiento & Ommo Gruppe \\
Jducación por el movimiento & José Maria Cagigal \\
Cultura física & Lean Le Boulch \\
El movimiento como acción & Kurt Meinel \\
Cultura del movimiento & Bart J. Crum \\
& Herbert Haag \\
Deporte & Roland Maul \\
& Ommo Gruppe \\
Conducta motriz & Voiger Ritner \\
Acción motriz & Pierre Parlebas \\
Experiencia corporal & Jean Barreau y Jean Morne
\end{tabular}

Para justificar el desarrollo de este trabajo, en que se quiere cooperar en develar si preservamos la corriente biologicista o ingresamos al desarrollo de todas las dimensiones de un ser humano físicamente bien educado, debemos reflexionar sobre aspectos sociales, políticos, éticos y estéticos de la enseñanza de la educación física que fortalecen a los futuros profesionales, pues su papel será innovar en la educación con capacidad reflexiva y crítica, participar proactivamente en un Estado de corte comunitario y armonizar y liderar una sana convivencia desde su saber.

En esa dirección, Zagalaz (2001) amplía el espectro de las tendencias de la educación física que, desde sus posturas teóricas y subsecuente recontextualización didáctica, enriquecen nuestro debate en torno a la formación de profesores:

- Educación físico-deportiva: el cuerpo acrobático

- Educación psicomotriz: el cuerpo pensante

- Expresión corporal: el cuerpo comunicante

- Sociomotricidad

- Habilidades motrices básicas

- Multideportiva

- Alternativa

- Actividades en la naturaleza

- Actividad física y salud

- Turismo y deporte

\section{Objetivos}

Entre ese constante flujo de discursos, saberes, conocimientos y experiencias, encontrados en múltiples conferencias, escuelas de pensamiento e interpretación de los académicos, se pretendió en este trabajo que los alumnos matriculados en los décimos semestres y próximos 
a egresar del nuevo Proyecto Curricular de Educación Física, PCLEF, de la Universidad Pedagógica Nacional, colaboraran como sujetos de investigación un año antes de la presentación de las pruebas ECAES, con los objetivos de:

- Identificar sus concepciones epistemológicas sobre las variables objeto de estudio y objetivo, sistema educativo y organizaciones, características programáticas y fundamentación, factores económicos, influencias, enfoque y tendencias, pedagogía e investigación, cruzadas por las disciplinas-saberes educación física, recreación y deporte.

- Inferir desde los aportes de este proyecto investigativo de aula una incursión en las diversas perspectivas teóricas que avizoran algunos cambios en el objeto de estudio, la pedagogía y el currículo, que merecen la atención de los formadores de formadores.

\section{Población}

Los sujetos de la investigación provenientes de dos cursos de décimo semestre de la primera y segunda promoción, de 25 y 26 alumnos respectivamente -que posteriormente se graduó del nuevo proyecto curricular de educación física iniciado en 2000-, se distribuyeron en cinco grupos focales cada uno para surtir el procedimiento de la investigación. Coincidió que la Facultad de Educación Física abría simultáneamente las licenciaturas en Deporte y Recreación, luego de un gran trayecto en su elaboración y sustentación, lo cual representó otro reto epistemológico para el paradigma unificado que vivió su claustro de profesores históricamente.

\section{Metodología}

El presente estudio utilizó el método cualitativo de grupos focales (Morgan, 1984 y 1988), que permite al investigador y a los investigados comprometerse en el proceso de búsqueda. Aquí los sujetos investigados se involucraron en la interpretación de la información, su significado y sus valores interactuando con el investigador; en otras palabras, se trabajó en la investigación como praxis: lo investigado fue analizado desde la teoría crítica que está más comprometida en la construcción colectiva, de lo útil para la sociedad cuestionando malas distribuciones de poder, recursos o servicios.

Los estudiantes utilizaron competencias comunicativas en tres encuentros de dos horas. En las de carácter interpretativo confrontaron significados y demostraron la articulación de conceptos y teorías, utilizando sus sa- beres y experiencias adquiridas en los espacios académicos de las áreas humanística, pedagógica y disciplinar. En las de carácter argumentativo, expusieron sus opiniones, apoyados en literatura sobre legislación y declaraciones de organismos internacionales, como la Unesco, OMS, Tafisa, FIEP, ICHPERD-SD, etc. En las de carácter propositivo, asumieron posturas constructivas y presentaron alternativas ante una buena diversidad de variables de la educación física, el deporte y la recreación, que se categorizaron para efectos del presente estudio.

\section{Resultados}

Posteriormente el autor de este proyecto y sus alumnos interpretaron, sistematizaron y elaboraron matrices para tabular la información obtenida en el proyecto de docencia-investigación educativa. Al procesar los datos se produjeron los siguientes resultados:

- Objeto de estudio y objetivo. Los sujetos denotaron su conocimiento e interés por el concepto experiencia corporal que básicamente da sustentación al currículo, también consideraron la opción de integralidad del cuerpo en el caso de la educación física como su principal objetivo. Para el deporte, dividieron sus opiniones entre el hombre en competencia y el deporte como fenómeno social, es decir, con énfasis en lo instrumental y el mercado, con objetivos de formación de habilidades motrices con fines preferentemente competitivos. En cuanto al objeto de estudio de la recreación, las respuestas apuntaron a que el ocio y la lúdica como conceptos son más importantes que el activismo recreativo, aunque el objetivo central es el aprovechamiento del tiempo libre para mejorar la calidad de vida.

- Sistema educativo y organizaciones. Los estudiantes creen que la escuela y las instituciones educativas, que son de la educación formal, deben desarrollar la educación física, pero también la estiman en los sectores no formales e informales, en parte debido a ciertos desarrollos de la educación física como movimiento popular; por ejemplo, el caso de la ciclovía. En la disciplina del deporte también dieron clara muestra de conocer las organizaciones, como las escuelas de formación, los clubes, las ligas, las federaciones que fomentan el deporte competitivo y, de alguna manera promocionan el deporte en los sectores populares a través de los eventos interbarriales e intercolegiados, cubriendo así los sectores formal, informal y no formal. En la disciplina de la recreación, los estudiantes 
optaron por señalar los sectores mencionados anteriormente donde se realizan las prácticas recreativas, pero también las ubicaron en entidades privadas, como cajas de compensación y específicamente en el IDRD, por su acción ampliamente reconocida a favor de las actividades recreativas y culturales de gran convocatoria.

- Enfoque y tendencias. La educación física fue tildada por los estudiantes con un sello humanista que desarrolla los ámbitos humanos y se orienta hacia los valores, salud y desarrollo integral de los individuos. Consideraron que el deporte está enfocado hacia el rendimiento del ser humano por buscar el perfeccionamiento de las técnicas, y su tendencia es la superación del deportista. Un enfoque socioantropológico se otorgó a la recreación, y aunque su tendencia es al aprovechamiento del tiempo libre, los sujetos enfatizaron la importancia de la interacción e integración de los individuos en su práctica.

- Pedagogía e investigación. En la educación física, los sujetos privilegiaron la pedagogía crítica sobre la tradicional y el modelo didáctico de Mosston que plantea como estilos de aprendizaje desde el comando directo hasta el desarrollo de autonomía. En cuanto a investigación, ponderaron con mayor peso el enfoque crítico social y la educativa de aula-cancha.

En el deporte señalaron que la pedagogía tradicional sigue siendo la favorita por su aproximación conductista. El modelo didáctico comando directo y asignación de tareas son los de mayor utilización. La investigación empírico-analítica y de laboratorio son las más reconocidas en ese ámbito por su labor en explicar, predecir y controlar el desempeño de los deportistas.

La recreación posibilita una reflexión desde la pedagogía social y de liberación para proveer a la sociedad en especial aquellas carentes de descanso, desarrollo y diversión. También se indicó que la pedagogía del ocio, con su modelo didáctico basado en la animación social, tiende a la solución de problemas y al desarrollo de la participación y autonomía en el disfrute del tiempo libre.

La investigación crítico-social y la hermenéutica son necesarias para comprender los fenómenos socioculturales de la práctica recreativa, aunque los enfoques positivistas son comunes de acuerdo con el contexto, sobre todo en la recreación como "entretenimiento".

- Características programáticas y fundamentación. Los participantes en el estudio adujeron que el currículo dirigido a niños en edad escolar es la máxima expresión en la educación física, que debe recibir las mayores contribuciones desde la pedagogía, la didáctica y la motricidad. En el deporte, el plan de entrenamiento para talentos seleccionados es la estrategia de intervención mediante el dominio de las ciencias biológicas sobre las humanas, además del desarrollo tecnológico. En la recreación, la intervención orientada a todo tipo de población que participa masiva, libre y espontáneamente, se hace mediante programación de actividades fundamentadas en los conocimientos y habilidades que el juego y sus dinámicas tienen, en la lúdica social.

- Factores económicos. Señalaron los estudiantes que la educación física está de acuerdo con el estrato socioeconómico, pues reviste unos costos fijos en equipos e instalaciones que difícilmente son cubiertos en su totalidad por las instituciones. El deporte es, según los investigados, una actividad de altos costos en adquisición de equipos de permanente actualización y construcción de escenarios de especificaciones muy precisas. En relación con la recreación, creen que a pesar de sus dimensiones masivas y de inclusión, los equipos son de fácil adquisición y los espacios se adecuan sin dificultad, ya que son comunitarios, urbanos o rurales, pues pertenecen a la comunidad, a la urbe y al campo.

- Influencias. En la actualidad la educación física está influenciada por factores socioeconómicos y políticos neoliberales, que han reducido las plazas de profesores en básica primaria para aumentar la cobertura. En el deporte, la influencia es muy parecida, pero más "desviada" por ser un espectáculo para las masas y un "producto de alta comercialización" que ha traído problemas de violencia, dopaje, sobornos, etc. La recreación es una expresión genuina de la gente en su afán de utilizar su tiempo libre en forma agradable. No obstante, influencias comerciales la han elitizado, perdiendo la posibilidad de subsidio e inclusión de las mayorías.

\section{Discusión}

Los resultados obtenidos en el desarrollo de este proyecto, sobre todo en el proceso para llegar a ellos, ponen de manifiesto, las siguientes consideraciones para abordar el segundo objetivo:

- Objetos de estudios dispersos. Como se observa, la concepción de diversos objetos de estudio en la educación física, el deporte y la recreación como disciplinas o saberes, se refleja en las diferentes carreras, caracterizadas por "diversas formas de conocimiento, 
que sirven para cuestionar, encontrar soluciones a los aspectos y problemas de la conducta motriz", como señala Rodríguez (1994).

En la formación de maestros y profesionales en los ámbitos nacional e internacional, asistimos a un modelo heterónomo configurado desde diferentes corrientes históricas y epistemológicas, constantes cambios políticos, reformas en la normatividad y legislación, y ubicación de las carreras en diferentes dependencias académicoadministrativas de las universidades. En consecuencia surgen estos interrogantes:

¿Contempla la educación física la recreación y el deporte en sus desarrollos teóricos y prácticos?

¿Considera el deporte a la ejercitación y la recreación dentro de sus desarrollos en el entrenamiento?

¿Estima la recreación la ejercitación y el deporte recreativo dentro de sus actividades?

¿Es la pedagogía la base integradora de la educación física, el deporte y la recreación?

- ¿Es la cultura física deporte, educación física, recreación, cultura física terapéutica y otras manifestaciones de la actividad física?

- ¿Recoge la actividad física al deporte, la educación física, la recreación activa en sus contenidos?

¿Es la actividad física simplemente cualquier movimiento corporal voluntario producido por los músculos esqueléticos para gastar energía y promover un estilo de vida saludable y activo?

Diversas pedagogías. Jaramillo (1997) anota que toda práctica educativa, en el pasado o en el presente, ha correspondido a determinada concepción pedagógica que la ha guiado, y que esta concepción haya sido consciente o inconscientemente asumida por los actores educativos es una referencia valiosa por cuanto las políticas neoliberales dan a la pedagogía un tinte reduccionista en torno a la disciplina, la ciencia y la tecnología.

Los resultados obtenidos en este estudio permiten reflexionar en la búsqueda del saber pedagógico, pues podríamos agrupar en estas tres tendencias los aportes de los estudiantes.

La educación física está saliendo a pasos agigantados de la pedagogía del desempeño, que Siedentop (1987) define como instrumentalismo pedagógico cuya didáctica se basa en procesos conductistas en desarrollo de conocimientos y evidencias de las áreas biomédica y motriz-deportiva. Al respecto, Bolívar (1998) afirma que "paulatinamente se ha ido comprendiendo que la educación física no es una actividad instrumental enca- minada a la competencia deportiva, sino una pedagogía que, desde su especificidad motriz y corpórea, ofrece a los educandos una experiencia lúdico-emocional, orientada al desarrollo humano armónico".

El deporte como práctica instrumentalista está cuestionado desde la pedagogía crítica que enfatiza en los paradigmas de liberación, diálogo, práctica y crítica, relacionadas con la ética, la moral, lo político (Giroux, 1988, 1990). Kirk (1986), en esa misma línea, tildado por algunos como antiepistemólogo, cree que la pedagogía debe rechazar los objetivos, y por tanto la planificación, el entrenamiento y las pruebas deportivas motrices, pues contribuyen a un dualismo que separa dos dimensiones del cuerpo.

En el proceso de consolidación de las respuestas de los sujetos sobre los objetivos de la educación física, el deporte y la recreación, se observa cierta inclinación por la pedagogía posmoderna, que cuestiona el pensamiento positivista y el crítico, buscando la intersujetividad para dar respuesta a las condiciones sociales, culturales y económicas que caracterizan el desarrollo del ser humano. Para Lyotard (1984), la relación entre sujeto-objeto de conocimiento se parece cada vez más a la relación proveedor-usuario o vendedor-consumidor, porque el conocimiento se produce y luego ser vendido, y luego consumido y transformado, perdiendo así su verdadero ser. Esta tesis causó gran controversia entre el estudiantado, pues adujeron que la educación física, el deporte y la recreación no se consideran un servicio, ni un producto, cuando se trata de educación.

\section{- Tensiones entre currícullo integrado, tradicional e} híbrido. Luego de analizar las teorías técnica, crítica y práctica sobre currículo, los sujetos registraron que el objeto de estudio para la educación física debe ser producto de un currículo integrado o alternativo, como lo señala López (1975), que asuma una posición crítica frente a las estructuras curriculares asignaturistas, pues estas no permiten expresar una concepción diferente en relación con el saber, con la relación profesor-alumno, con el papel de estudiante, con el papel del profesor, con el proceso evaluativo y con el proceso investigativo.

Esta circunstancia ha sido tenida en cuenta, fraccionadamente, por varios programas que articulan su diseño curricular en los núcleos problémicos o estrategias curriculares interdisciplinarias que acogen la investigación y la extensión en un supuesto currículo integrado o por el asignaturismo del currículo tradicional, que tiende a una acción operativa e instrumental en un proceso 
acrílico y rutinario, en detrimento de la reflexión. Por tanto, es necesario revisar la teoría crítica de la sociedad que comparte Stephen Kemmis (1986), quien afirma que "las teorías curriculares son teorías sociales, no sólo porque reflejan la historia de las sociedades en que surgen, sino también en el sentido de que están vinculadas con posiciones sobre el cambio social y en particular con el papel de la educación en la reproducción o transformación de la sociedad".

\section{Conclusiones}

Es esencial la vinculación de los estudiantes próximos a graduarse en la fijación de sus propias competencias; por consiguiente, se les puede admitir en el proceso de levantamiento de información para los ECAES, pues es justo y fiable preguntarles lo que saben y no lo que ignoran. Su inmersión en el proceso de preparación para esos exámenes en las instituciones de educación superior contribuirá a la definición de la naturaleza epistémica y al desarrollo de marcos conceptuales de su programa de formación, brindará una excelente retroalimentación.

Los resultados expuestos en este artículo convidan a una aproximación conceptual de los programas universitarios para establecer, además de las competencias, acuerdos sobre objetos de estudio, elementos pedagógicos y didácticos, investigación, diseño curricular etc., con base en el Decreto 2566 de 2003, que establece las condiciones mínimas y demás requisitos para desarrollar los programas de educación superior. El autor de

\section{Referencias}

Álvarez, A. (2003). Acreditar, certificar y evaluar: tres procesos distintos y un solo Estado verdadero. Pedagogía y saberes, 18, 45-52.

Bahamón, P. (2007). Las competencias: acerca de su dimensión pedagógica y humanística. Memorias II Congreso Ascofade. Medellín: Farive S. A.

Bolívar, C. (1998). Referente teórico de la educación física para el desarrollo humano. Revista Kinesis, 29.

Calderón, A. (2007) Concepciones de los profesores de educación física sobre el deporte: de la hegemonía a utopía. Lúdica Pedagógica, 84-95.

Giroux, H. (1990). Rethinking the boundaries of educational discourse. Modernism, postmodernism, and feminism. College Literature, $17(2-3), 1-15$.

Giroux, H. and Simon, R. (1988). Critical pedagogy and the politics of popular culture. Unpublished manuscript.

Ibarra, O. A. (2004). Carta abierta del rector a la comunidad universitaria. Serie Reflexiones 5, 3. Bogotá: Universidad Pedagógica Nacional.

Jaramillo, J. (1997). Procesos pedagógicos educación física, escuela y sociedad. Revista Kinesis, 21, 23.

Kemmis. S. (1986). El curriculum. Madrid: Ediciones Morata. este trabajo ha confirmado, en su labor de evaluación de programas en algunas universidades del país, que:

- El apogeo de la producción intelectual, incrementada, en los últimos años, merece revisarse para mantener la unidad de los campos de la educación física, el deporte y la recreación, o para separarlos, aunque sin perder conexiones

- La aparición de redes como Arcofader, Amedco, así como la labor esforzada y tradicional de la Asociación de Profesores de Educación Física y de otras de egresados de diferentes programas está logrando cambios significativos y retroalimentación a la gestión académica.

- Se atestigua un progreso en el diseño y la estructuración curricular, en la creación de grupos, proyectos y líneas de investigación y en el impuso a proyectos de extensión a la comunidad respaldados por convenios interinstitucionales.

- Hay profusión de programas de pregrado en el país; la titulación de posgrados se amplía cada año y es un hecho el recorrido hacia lo científico a través de la interdisciplinaridad de la comunidad académica.

- Así encontraremos un espacio epistemológico para la pedagogía, como lo manifiesta Ibarra (2004), con el propósito de "definir los elementos esenciales del saber específico propio del maestro y de sus relaciones con la ciencia y la tecnología, con la ética y la cultura, en un proceso, si se quiere, ordenado intencionalmente, pero no determinado ni estructurado para el adiestramiento". (D)
Kirk, D. (1986). Beyond the limits of theoretical discourse in teacher education: towards a critical pedagogy. Teaching and Teacher Education, 2 (2), 155-167.

López, N. (1995). La reestructuración curricular de la Educación Superior. Icfes, Presencia, 74.

Lyotard, J. (1984). The postmodern condition: a report on knowledge. Minneapolis: University of Minnesota Press.

Ministerio de Educación Nacional. (2000). Educación física, recreación y deportes, Bogotá, 34-35.

Morgan, D. L. \& Spanish, M.T. (1984). Focus groups: a new tool for qualitative research. Qualitative Sociology, 3, 253-270.

Morgan, D. L. (1988). Focus groups as qualitative research. Newbury Park, CA: Sage.

Rees, \& M. Pieron (Eds.). Myths, models, and methods in sport pedagogy, 79-86. Champaign, IL: Human Kinetics

Quiceno, H. (2006). El maestro: del oficio a la profesión. Miradas críticas. Separata, Educación y Pedagogía, Medellín.

Siedentop, D. (1987). The theory and practice of sport education. In G. Barrette, R. Feingold, R.

Rodríguez, J. (1994). Deporte y ciencia. Barcelona: INDE.

Zagalaz, M. (2001). Corrientes y tendencias de la educación física. Barcelona: Inde Publicaciones, 73. 